\title{
Evaluación financiera de la industria del cemento, cal y yeso en Colombia (2014-2019)*
}

[Artículos]

\author{
Jorge Alberto Rivera Godoy** \\ Carlos Andrés Trejos Pérez ${ }^{* * *}$ \\ José Luis Quevedo Suárez****
}

Recibido: 23 de abril de 2021

Aprobado: 03 de junio de 2021

Citar como:

Rivera, J., Trejos, C. y Quevedo, J., (2021). Evaluación financiera de la industria del cemento, cal y yeso en Colombia (2014-2019). Revista

Activos, 19(1). https://doi.org/10.15332/25005278.6687

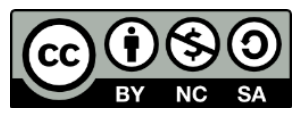

\section{Resumen}

La investigación reportada en el presente artículo tiene como objetivo evaluar el desempeño financiero de la industria del cemento, cal y yeso

\footnotetext{
* Artículo resultado de la línea de investigación de "Evaluación del desempeño financiero de empresas del sector real en Colombia", que adelanta el Grupo de Investigación en Generación de Valor Económico de la Universidad del Valle (categoría C de Colciencias).

** Profesor titular, Universidad del Valle. Doctor distinguido "Cum Laude" en Ciencias Económicas y Empresariales, Universidad Autónoma de Madrid. Coordinador del grupo de investigación en Generación de Valor Económico (GIGVE) de la Universidad del Valle. Correo electrónico: jorge.rivera@correounivalle.edu.co; ORCID: http://orcid.org/0000-0003-2319-1674; CvLAC http://scienti.colciencias.gov.co:8081/cvlac/visualizador/generarCurriculoCv.do?cod rh=0000517 $\underline{305}$

${ }_{* * *}$ Estudiante de Contaduría Pública de la Universidad del Valle, Cali, Colombia. Correo electrónico: trejos.carlos@correounivalle.edu.co; ORCID: http://orcid.org/0000-0002-9819-8515 **** Estudiante de Contaduría Pública de la Universidad del Valle, Cali, Colombia. Correo electrónico: jose.quevedo@correounivalle.edu.co; ORCID: http://orcid.org/0000-0003-3102$\underline{0701}$
}

Revista Activos

ISSN: 0124-5805 | e-ISSN: 2500-5278 | DOI: https://doi.org/10.15332/25005278

Vol. 19 N.o 1 | enero-junio de 2021 
en Colombia en el lapso 2014-2019. Se empleó como metodología el análisis estático y de tendencias de indicadores contables y de gestión de valor que miden su crecimiento, eficiencia, eficacia, efectividad, valor económico agregado (EVA) y valor de mercado agregado (VMA). Se encontró que este sector aumenta sus ventas y activos, pero reduce su utilidad neta; es efectivo en el logro de utilidades y sigue la orientación cambiante de la eficacia en el control de erogaciones, que es amplificada por un apalancamiento financiero positivo. No obstante, destruye el EVA en los últimos cuatro años, por causa del deterioro del rendimiento después de impuestos del activo neto operacional, que fue superado por el costo de capital. Estos hallazgos pueden complementarse con estudios para grupos homogéneos en edad, tamaño y naturaleza jurídica.

Palabras clave: valor económico agregado (EVA), valor de mercado agregado, desempeño financiero, indicadores financieros de eficiencia, indicadores financieros de efectividad, indicadores financieros de eficacia, sector cemento, cal y yeso, manufacturas, Colombia.

Clasificación JEL: G10, M40, L61.

\section{Financial evaluation of the cement, lime and gypsum industry in Colombia (2014-2019)}

\section{Abstract}

The research reported in this article aims to evaluate the financial performance of the cement, lime and gypsum industry in Colombia in the 2014-2019 period. The static and trend analysis of accounting and value management indicators that measure its growth, efficiency, efficacy, effectiveness, economic value added (EVA) and market value added (VMA) was used as a methodology. It is found that this sector increases its sales and assets but reduces its net profit; it is effective in obtaining profits, following the changing direction of efficacy in controlling expenditures, which is amplified by positive financial leverage. However, it destroys EVA in the last four years, due to the deterioration of the 
after-tax return on net operating assets, which was exceeded by the cost of capital. These findings can be complemented with studies for homogeneous groups in age, size and legal nature.

Keywords: economic value added (EVA), market value added, financial performance, financial indicators of efficiency, financial indicators of effectiveness, financial indicators of efficacy, cement, lime and gypsum sector, manufacturing, Colombia.

JEL classification: G10, M40, L61.

\section{Introducción}

La industria de cemento, cal y yeso hace parte de los sectores estratégicos de la economía colombiana porque aportan suministros vitales para el sector de la construcción, considerado como uno de los principales motores de la economía colombiana. En el periodo 2014-2019, la producción y despachos han sufrido altibajos, dado el aumento en la demanda del segmento de construcción de obras civiles, pero no así del segmento inmobiliario, lo que ha incidido en el decrecimiento del sector de la construcción (Sectorial, 2020). No obstante, en promedio ha mantenido 32 establecimientos que han generado 3999 empleos y su producción representa el 0,92 \% del sector manufacturero (Departamento Administrativo Nacional de Estadística [DANE], 2020a; 2020b). A pesar de que existen algunos estudios sobre su producción, demanda, empleabilidad, comercio internacional y su impacto ambiental, su desempeño financiero ha sido poco investigado. Se ha abordado mayoritariamente desde trabajos de grado y tesis de maestría en Colombia, donde aún queda como interrogante conocer cuál ha sido su efectividad para producir utilidades sobre la inversión y si el propósito financiero de crear valor económico se ha cumplido. El objetivo principal de esta investigación apunta a resolver estos enigmas. 
Este estudio aplica como metodología el análisis financiero de indicadores en el periodo 2014-2019 con un enfoque cuantitativo. Estos son calculados a partir de datos contables y de mercado, que permiten analizar el crecimiento, la eficiencia en el empleo de los activos, la eficacia en la administración de costos y gastos, la estructura de capital y el costo de capital, como factores que inciden sobre la efectividad en el logro de utilidades y la creación de valor económico (Dumrauf, 2017; Anaya, 2018; Rivera, 2017; Ross et al., 2019; Stewart 2000).

Las ventas y los activos crecen, mientras que la utilidad neta disminuye, en tanto todos fluctúan en diferentes frecuencias. Asimismo, se logran rentabilidades contables para los socios, lo cual está influenciado esencialmente por el comportamiento de la eficacia en el monitoreo de costos y gastos, que es ampliada por la utilización de un positivo apalancamiento financiero. Sin embargo, este sector destruye EVA entre el 2016 y el 2019, lo que dejó como saldo un VMA negativo en el sexenio. Estos hallazgos son comparados con las grandes empresas de este sector en Colombia y con el sector afín en Estados Unidos de América. Hay un comportamiento semejante en las ventas y utilidad neta entre los dos grupos colombianos, pero es más efectivo todo el sector cementero colombiano, dada su mayor eficacia en el control de costos y gastos totales. Esto, en parte, contrarrestó su menor eficiencia en la utilización de los activos y su menor uso de una palanca financiera positiva. No obstante, el sector afín estadounidense fue más efectivo que las dos agrupaciones colombianas. En la revisión del valor económico agregado, se encuentra que el sector cementero colombiano destruye valor en el sexenio porque, a pesar de lograr una rentabilidad después de impuestos sobre los activos netos operacionales, no fue suficiente para cubrir el costo de los recursos financieros. Caso contrario sucedió en el sector análogo estadounidense, donde se creó valor en el periodo. 
El escrito se organiza de la siguiente forma: primero, se trata el marco teórico, donde se definen los indicadores financieros pertinentes para este análisis; segundo, se plantea la metodología, exponiendo su enfoque, método, alcance y fuentes de información para desarrollar la investigación; tercero, se caracteriza la industria de cemento, cal y yeso en Colombia, y se presentan los resultados de los principales indicadores de desempeño financiero de otros sectores semejantes en el país y en Estados Unidos de América, que sirven como referentes, y se describe el desempeño financiero de esta industria en Colombia; cuarto, se analizan y comparan los resultados, y, quinto, se presentan las conclusiones.

\section{Marco teórico}

La información contable es empleada frecuentemente por organizaciones (sectores económicos) para medir y examinar su desempeño financiero, ya que con ella se pueden establecer indicadores que permiten la evaluación de diferentes actividades financieras. Entre ellos, se tienen los que cuantifican el crecimiento, la eficiencia, la eficacia y la efectividad. Los indicadores de crecimiento miden el desarrollo del sector conforme a los activos, ventas y utilidad neta (Dumrauf, 2017).

"La eficiencia con la cual una empresa utiliza sus recursos, en especial los activos operacionales, según la velocidad de recuperación del dinero invertido en cada uno de los mismos" (Anaya, 2018, p. 223) es considerada por los indicadores de rotación de activos. Así, sobresale la rotación de cartera, la rotación de inventarios, la rotación de activos fijos, la rotación de activos netos operacionales y la rotación de los activos totales (Rivera, 2017). Para determinarlos, se calcula el cociente de las ventas sobre el promedio de la categoría de activo, excepto en la de inventarios donde se toma el costo de ventas como numerador porque los inventarios están 
asentados a valor de costo. El cociente indica la cantidad de veces que ha rotado el activo en el lapso que tuvieron lugar las ventas.

La eficacia en el control de los costos y gastos de las compañías y su impacto sobre la utilidad son considerados por los márgenes de utilidad como, por ejemplo, el margen de utilidad bruta, el margen de utilidad operacional, el margen de utilidad operacional después de impuestos y el margen de utilidad neta (Rivera, 2017). Estos indicadores surgen del cociente de cada una de las utilidades sobre las ventas. Este cociente denota la utilidad que dejan las ventas, que puede ser expresado en unidades monetarias o en forma porcentual.

La efectividad en la obtención de beneficios económicos para los inversores o propietarios, por la inversión realizada, se cuantifica por medio de los siguientes indicadores: el primero, por la rentabilidad del activo (ROA), y, el segundo, por la rentabilidad del patrimonio (ROE) ${ }^{1}$ (Ross et al., 2019). El sistema Dupont muestra que el ROA “depende de dos factores: las ventas que la compañía genera a partir de sus activos (rotación de activos) y la ganancia que obtiene sobre cada dólar de ventas (margen de utilidad de operación)" (Brealey et al., 2015, p. 715) y se halla multiplicando estos dos componentes. Con el sistema Dupont ampliado, se establece que el ROE surge de tres factores: la rotación de activos, el margen de utilidad neta y el multiplicador del capital contable ${ }^{2}$ (Brigham y Ehrhardt, 2018). Dicho de otra forma, la efectividad revelada por el ROA surge del producto de la eficiencia por la eficacia, mientras que la efectividad medida por el ROE resulta del producto de la eficiencia, la eficacia y el apalancamiento financiero (Rivera, 2017).

\footnotetext{
${ }^{1} R O A=$ utilidad operacional / activo promedio $R O E$ = utilidad neta / patrimonio promedio

${ }^{2}$ El multiplicador de capital contable es una manera de determinar el apalancamiento financiero, que resulta de dividir el activo promedio sobre el patrimonio promedio.
} 
Los indicadores contables como el ROA, el ROE y la utilidad por acción (UPA) son muy usados para examinar el desempeño de las empresas, aunque son cuestionados por estar influenciados por las políticas contables adoptadas (Atrill, 2017), por estar expuestos a ser manipulados (Salaga et al., 2015), y por no considerar el riesgo ni el costo de capital propio (Stern y Willette, 2014). Esto ha provocado el aparecimiento de modelos de gestión basados en el valor que monitorean el desempeño financiero mediante un seguimiento de la utilidad residual, donde el EVA es el más conocido (Worthington y West, 2001).

El EVA es una utilidad residual que se deriva de la diferencia entre la utilidad operacional después de impuestos y el cargo por el empleo del capital (Stewart, 2000), como se muestra en la ecuación (1):

$$
\mathrm{EVA}_{\mathrm{t}}=\mathrm{UODI}_{\mathrm{t}}-\text { cargo de capital }
$$

donde $\mathrm{UODI}_{\mathrm{t}}$ es la utilidad operacional después de impuestos en el periodo t, y el cargo de capital en el periodo t es igual a:

$$
\text { Cargo de capital }=\left(\mathrm{ANO}_{\mathrm{t}}\right)\left(\mathrm{Ko}_{\mathrm{t}}\right)
$$

$\mathrm{ANO}_{\mathrm{t}}$ es el activo neto operacional del periodo. $\mathrm{El} \mathrm{ANO}_{\mathrm{t}}$ es la suma del capital de trabajo neto operativo $\mathrm{KTNO}_{\mathrm{t}} \mathrm{y}$ del activo fijo neto operacional $\mathrm{AFNO}_{\mathrm{t}}$ :

$$
\mathrm{ANO}_{\mathrm{t}}=\mathrm{KTNO}_{\mathrm{t}}+\mathrm{AFNO}_{\mathrm{t}}
$$

El $\mathrm{KTNO}_{\mathrm{t}}$ es la diferencia entre el activo circulante y el pasivo corriente sin costo explícito. El $\mathrm{AFNO}_{\mathrm{t}}$ es la diferencia entre los activos fijos operacionales y la depreciación.

$\mathrm{El} \mathrm{Ko} \mathrm{t}$ es el promedio ponderado del costo de capital en el periodo t; de acuerdo con Modigliani y Miller (1963, p. 441), se calcula así: 


$$
\mathrm{Ko}_{\mathrm{t}}=\mathrm{Ke}(1-\mathrm{L})+\mathrm{Ki}(1-\mathrm{t}) \mathrm{L}
$$

Ke es el costo del patrimonio3. L es la estructura de capital que resulta de dividir la deuda con costo explícito entre el ANO. Ki es el costo de la deuda, pero dado que los intereses tienen protección fiscal, el costo de la deuda quedaría reformulado como $\mathrm{Ki}(1-\mathrm{t})$, donde t es la tasa impositiva de la compañía.

Otra manera de hallar el EVA (Stewart, 2000) se expresa en la ecuación (5):

$$
\mathrm{EVA}=\left(\mathrm{ANO}_{\mathrm{t}}\right)\left[\left(\mathrm{UAII}_{\mathrm{t}}\right)(1-\mathrm{t}) /\left(\mathrm{ANO}_{\mathrm{t}}\right)-\left(\mathrm{Ko}_{\mathrm{t}}\right)\right]
$$

donde $\left(\mathrm{UAII}_{\mathrm{t}}\right)(1-\mathrm{t}) / \mathrm{ANO}_{\mathrm{t}}$ es la rentabilidad del activo neto operacional después de impuestos. A la diferencia entre la rentabilidad del activo neto operacional después de impuestos y el promedio ponderado del costo de capital, se le llama el porcentaje de utilidad o pérdida residual.

El indicador $\left(\mathrm{UAII}_{\mathrm{t}}\right)(1-\mathrm{t}) / \mathrm{ANO}_{\mathrm{t}}$ se puede descomponer en dos partes de manera semejante al sistema Dupont: $\left(\mathrm{UAII}_{\mathrm{t}}\right)(1-\mathrm{t}) /$ ventas $_{\mathrm{t}} \mathrm{y}$ ventas $\mathrm{t}_{\mathrm{t}} / \mathrm{ANO}_{\mathrm{t}}$. La primera corresponde al margen de utilidad operacional después de impuestos y la segunda a la rotación de activos netos operacionales.

A la sumatoria del valor actual del EVA de varios años se le denomina valor de mercado agregado VMA; y matemáticamente se expresa según la ecuación (6):

${ }^{3}$ Se acoge a la metodología del CAPM de Pure-Play presentada por Rivera y Alarcón (2012), para
calcular el Ke, debido a que la gran mayoría de las empresas que conforman este sector no cotizan en la bolsa de valores. 


$$
V M A=\sum_{j=1}^{j=n} \operatorname{EVA}_{j} /(1+K o j)^{j}
$$

\section{Metodología}

Para analizar el desempeño financiero del sector cemento, cal y yeso en Colombia en el periodo 2014-2019, y se siguió un enfoque cuantitativo de investigación, que permitió examinar los factores que repercutieron sobre su comportamiento. Se utilizó como método el análisis estático y de tendencias de indicadores contables (Gitman y Zutter, 2016) y de gestión del valor más adecuados para la evaluación financiera de compañías de una industria. Los hallazgos fueron confrontados con los de sectores pares del contorno nacional e internacional.

El crecimiento, la eficiencia, la eficacia y la efectividad fueron los indicadores contables escogidos, mientras que el EVA y el VMA fueron los indicadores de administración del valor seleccionados.

Se examina la administración del valor con el EVA y sus inductores: UODI, ANO y Ko; con el sistema Dupont de la razón UODI/ANO y con el VMA. Los indicadores contables y de medición de la creación de valor económico se calcularon con base en los estados financieros de las empresas de la industria del cemento, cal y yeso en Colombia en el periodo 2014-2019, tomados de EMIS Professional (2020), de donde se obtuvo información de 18 empresas para el 2013, 2015 y 2016; 20 empresas para 2014 y 2017, y 21 empresas para el 2018 y 2019. La razón social y su número de identificación tributaria se presentan en el anexo 1.

Se realizaron comparativos con sectores semejantes en el medio nacional e internacional mediante el cálculo de indicadores de desempeño financiero 
con información contenida en la revista Dinero (2014, 2015, 2016, 2017, 2018, 2019, 2020) y en Damodaran (2020).

\section{Resultados}

En esta sección se describe el sector dedicado a la fabricación de cemento, cal y yeso en Colombia, se presentan dos estudios de referencia del desempeño financiero de sectores afines en el entorno nacional e internacional, y finalmente se evalúa dicho sector (para efectos de simplicidad se hace referencia al sector cemento o cementero).

\section{Caracterización del sector de fabricación de cemento, cal y yeso en Colombia}

De acuerdo con la clasificación industrial internacional uniforme (CIIU) las empresas dedicadas a esta actividad económica hacen parte de la clase 2394, que incluye:

- La fabricación de clinkers y cementos hidráulicos, incluidos cemento Portland, cemento aluminoso, cemento de escorias y cemento hipersulfatado.

- La fabricación de cal viva (caliza y dolomita calcinada).

- La fabricación de cal apagada.

- La fabricación de cal hidráulica.

- La fabricación de yesos a partir de yeso calcinado y sulfato de calcio.

- La fabricación de dolomita calcinada. (DANE, 2020c, p. 276)

En el periodo 2014-2019, los establecimientos colombianos dedicados a la fabricación de cemento, cal y yeso fueron, en promedio, alrededor de 32, lo 
que representó el 0,38 \% de la industria manufacturera. Fue significativa la reducción presentada en el 2015, pero, a partir de ahí, con variaciones menores en los siguientes años; de manera semejante se comportó el número de empleos generados por este sector, con un bajón importante en el 2015, pero luego fluctuó el resto de años de manera más moderada. El promedio del personal ocupado fue de 3999, que equivale al o,6 \% de empleabilidad de la industria manufacturera. La producción promedio de la industria de cemento, cal y yeso fue de $\$ 1312$ millones de pesos (0,92 \% de la industria nacional) con una gran caída del 2014 al 2016, y posteriormente oscila con crestas menores (tabla 1).

Tabla 1. Fabricación de cemento, cal y yeso: establecimientos, empleos y producción con relación al sector manufacturero en Colombia

\begin{tabular}{|c|r|r|r|r|r|r|r|}
\hline & $\mathbf{2 0 1 4}$ & $\mathbf{2 0 1 5}$ & $\mathbf{2 0 1 6}$ & $\mathbf{2 0 1 7}$ & $\mathbf{2 0 1 8}$ & $\mathbf{2 0 1 9}$ & Promedio \\
\hline N.o establecimientos & 39 & 29 & 33 & 30 & 31 & 30 & 32 \\
\hline \% de establecimientos & 0,43 & 0,32 & 0,39 & 0,37 & 0,39 & 0,39 & 0,38 \\
\hline N.o empleos & 5259 & 3478 & 3948 & 3661 & 3885 & 3762 & 3999 \\
\hline \% de empleos & 0,77 & 0,49 & 0,54 & 0,51 & 0,55 & 0,53 & 0,60 \\
\hline Producción MMM\$ & 4754 & 652 & 574 & 589 & 541 & 764 & 1312 \\
\hline \% producción & $\mathbf{2 , 2 4}$ & $\mathbf{0 , 2 9}$ & $\mathbf{0 , 2 4}$ & $\mathbf{0 , 2 4}$ & $\mathbf{0 , 2 1}$ & $\mathbf{0 , 2 8}$ & $\mathbf{0 , 9 2}$ \\
\hline
\end{tabular}

Nota: cifra monetaria en miles de millones de pesos (MMM\$).

Fuente: elaboración propia con base en DANE (2020a y 2020b).

El sector cementero ha estado ligado con el dinamismo del sector de la construcción, que en el periodo 2014-2019 ha venido disminuyendo su crecimiento en el primer trienio y decreciendo en el segundo trienio. En los tres primeros pasó de un 8,9 \% en el 2014 a un 3,6 \% en 2016, y los siguientes años fue de $-2,0 \%$ en el 2017, $-1,3 \%$ en el 2018 y $-1,9 \%$ en el 2019. En los primeros años la reducción estuvo notoriamente jalonada por la merma en las obras de infraestructura que pasaron de 13,2 \% en el 2014 
a -2,6 \% en el 2016, mientras que en el segundo trienio el decrecimiento lo marcó la contracción de la construcción inmobiliaria (tabla 2).

Tabla 2. Variación del PIB de la construcción (\%)

\begin{tabular}{|c|c|c|c|c|c|c|}
\hline & 2014 & 2015 & 2016 & 2017 & 2018 & 2019 \\
\hline Construcción & 8,9 & 6,3 & 3,6 & $-2,0$ & $-1,3$ & $-1,9$ \\
\hline $\begin{array}{l}\text { Construcción de edificaciones residenciales y } \\
\text { no residenciales }\end{array}$ & 7,0 & 6,4 & 6,0 & $-5,2$ & $-0,3$ & $-8,4$ \\
\hline $\begin{array}{l}\text { Construcción de carreteras y vías de } \\
\text { ferrocarril, de proyectos de servicio público y } \\
\text { de otras obras de ingeniería civil }\end{array}$ & 13,2 & 6,7 & $-2,6$ & 6,5 & $-2,4$ & 11,2 \\
\hline
\end{tabular}

Fuente: elaboración propia con base en DANE (2021).

Es indudable que las vías 4G, el proyecto de Metro en Bogotá y el desarrollo de poblaciones en Colombia ha sido determinante para el crecimiento del sector cementero, que en la última década ha visto la llegada de seis nuevas compañías, que lo convierte en uno los países con mayor número de fabricantes de la región. Sin embargo, han surgido algunos obstáculos por temas ambientales, de comunidades y de cierres financieros en obras civiles, temores del sistema financiero a la hora de invertir y poco dinamismo de la vivienda no VIS que han retrasado las obras y provocado una sobreoferta (Neira, 2019).

\section{Estudios de referencia del desempeño financiero del sector cemento}

En la tabla 3 se han determinado y clasificado los indicadores de desempeño financiero para una media de 25 compañías más grandes del sector cemento y concreto en Colombia (GCC) en el sexenio 2014-2019, que reporta en una edición especial anual la revista Dinero, bajo el título: "Ranking de las 5000 empresas más grandes de Colombia".

Las ventas, los activos, el patrimonio y la utilidad crecieron hasta el 2016, pero en los años siguientes las ventas cayeron, mientras que los otros tres 
indicadores oscilaron de manera semejante. Los promedios en el sexenio fueron: ventas \$ 677.526 MM, activos \$ 1.610.340 MM, patrimonio \$ 893.894 MM y utilidad neta \$ 40.970 MM; los valores más altos se presentaron en 2016 y los más bajos en el 2014 para las ventas y activos, y en el 2017 para el patrimonio y utilidad neta (parte a de la tabla 3). 
Tabla 3. Indicadores contables promedio de las GCC

\begin{tabular}{|c|c|c|c|c|c|c|c|}
\hline Indicador & 2014 & 2015 & 2016 & 2017 & 2018 & 2019 & promedio \\
\hline \multicolumn{8}{|c|}{ a. Crecimiento (MM\$) } \\
\hline Ventas & 571.805 & 729.507 & 819.738 & 675.034 & 639.132 & 629.942 & 677.526 \\
\hline Activos & 1.1.542.319 & 1.682 .712 & 1.943 .563 & 1.336 .449 & 1.652 .626 & 1.504 .373 & 1.610 .340 \\
\hline Patrimonio & 931.474 & 970.846 & 1.088 .334 & 661.793 & 916.352 & 794.567 & 893.894 \\
\hline Utilidad neta & 41.014 & 79.272 & 85.467 & 1.094 & 24.152 & 14.823 & 40.970 \\
\hline \multicolumn{8}{|c|}{ b. Eficiencia (veces) } \\
\hline Rotación de activos ${ }^{1}$ & 0,4 & 0,5 & 0,5 & 0,4 & 0,4 & 0,4 & 0,4 \\
\hline \multicolumn{8}{|c|}{ c. Eficacia (\%) } \\
\hline Utilidad neta/ventas ${ }^{2}$ & 7,2 & 10,9 & 10,4 & 0,2 & 3,8 & 2,4 & 5,8 \\
\hline \multicolumn{8}{|c|}{ d. Apalancamiento financiero (\%) } \\
\hline Activo/ patrimonio ${ }^{3}$ & 158,7 & 169,5 & 176,1 & 187,4 & 189,4 & 184,5 & 177,6 \\
\hline \multicolumn{8}{|c|}{ e. Efectividad (\%) } \\
\hline $\mathrm{ROE}^{4}$ & 4,8 & 8,3 & 8,3 & 0,1 & 3,1 & 1,7 & 4,4 \\
\hline \multicolumn{8}{|c|}{ f. Número de GCC } \\
\hline N. Empresas & 23 & 25 & 23 & 21 & 28 & 31 & 25 \\
\hline
\end{tabular}

Nota: $\mathrm{MM} \$$ significa cantidad en millones de COP.

$$
{ }^{1} \sigma=0,02^{2} \sigma=4,4^{3} \sigma=11,9{ }^{4} \sigma=3,1
$$

Fuente: elaboración propia, con información de la revista Dinero (2014, 2015, 2016, 2017, 2018, 2019 y 2020$).$ 
La rotación de activos aumentó de 0,4 veces en el 2014 a 0,5 veces en el 2015 y 2016, pero descendió a 0,4 veces en el 2017, y se mantuvo así hasta el final del periodo. Lo anterior dejó como resultado un promedio de eficiencia en el uso de los activos de o,4 veces al año (parte b de la tabla 3). El margen de utilidad neta varió anualmente, salvo entre 2016-2017, cuando se redujo; su promedio en el sexenio fue de 5,8 \%; la mayor eficacia tuvo lugar en el 2015 (10,9\%), pero dos años después pasó a ser la menor del periodo (o,2 \%), como se aprecia en la parte c de la tabla 3 . La relación activo/patrimonio creció hasta el penúltimo año, pero al final se redujo; su promedio fue de 177,6 \%, y pasó de un apalancamiento financiero inferior en el 2014 (158,7\%) al mayor del periodo en el 2018 (189,4 \%), como se muestra en la parte d de la tabla 3.

El rendimiento del patrimonio fluctuó en casi todos los años cerca de un promedio de 4,4\%, con tendencia similar a la eficacia, solo interrumpido en el 2016. Después de pasar dos años consecutivos con los mayores indicadores de efectividad: 2015 y 2016 con 8,3 \%, pasó a su menor indicador del periodo en el 2017 con 0,1 \%, lo que confirma el impacto que tuvo la eficacia en la gestión de costos y gastos sobre la efectividad en lograr beneficios para los propietarios. La eficiencia en el uso de los activos también explica los cambios de la efectividad en estos tres años, pero no en los otros. Por su parte, el apalancamiento financiero no fluctúa, pero sí ejerce un efecto multiplicador positivo del margen de utilidad neta (tabla 3, sección e, c, d y b).

De Damodaran (2020) se consiguieron datos de un promedio de 57 compañías del sector suministros de construcción en los Estados Unidos de América (SCEUA) en el periodo 2014-2019, que permitió determinar los indicadores de desempeño financiero que se muestran en la tabla 4. 
Este sector estadounidense creó valor económico cada año, con tendencia a declinar hasta el 2016, pero luego aumentó hasta el 2019. El EVA promedio fue de $\$ 5542$ MMUS, y muestra su valor inferior en el 2016 (\$ 2021 MMUS) y su valor superior en el 2019 (\$10.212 MMUS). Este comportamiento sigue la orientación de la UODI, salvo en el 2015, y una dirección contraria al cargo de capital, excepto en el 2016 y 2018. La UODI promedio fue de \$ 14.135 MMUS, con una cuantía inferior en el 2016 (\$ 10.298 MMUS) y una suma mayor en el 2019 (\$ 18.444 MMUS), años en los que el EVA fue menor y mayor, respectivamente. El cargo de capital promedio fue de \$ 8593 MMUS, con un mayor importe en el 2015 (\$ 9560 MMUS), y un menor costo en el 2017 ( 7284 MMUS), lo que corrobora una mayor relación entre EVA y UODI.

Tabla 4. Indicadores promedio del sector suministros de construcción en EUA (SCEUA)

\begin{tabular}{|c|c|c|c|c|c|c|c|}
\hline Indicador & 2014 & 2015 & 2016 & 2017 & 2018 & 2019 & promedio \\
\hline EVA (MMUS\$) & 4.288 & 4.252 & 2.021 & 3.982 & 8.498 & 10.212 & 5.542 \\
\hline UODI (MMUS\$) & 13.317 & 13.812 & 10.298 & 11.266 & 17.673 & 18.444 & 14.135 \\
\hline $\begin{array}{l}\text { Cargo de capital } \\
\text { (MMUS\$) }\end{array}$ & 9.029 & 9.560 & 8.277 & 7.284 & 9.175 & 8.232 & 8.593 \\
\hline ANO (MMUS\$) & 107.259 & 113.386 & 109.008 & 106.402 & 104.777 & 115.413 & 109.374 \\
\hline Кo $(\%)^{1}$ & 8,4 & 8,4 & 7,6 & 6,8 & 8,8 & 7,1 & 7,9 \\
\hline UODI/ANO (\%) ${ }^{2}$ & 12,4 & 12,2 & 9,4 & 10,6 & 16,9 & 16,0 & 12,9 \\
\hline $\mathrm{Ke}(\%)^{3}$ & 11,4 & 12,2 & 9,9 & 8,1 & 11,3 & 9,0 & 10,3 \\
\hline $\begin{array}{l}\text { VMA a 1-1-2014 } \\
\text { (MMUS\$) }\end{array}$ & 24.384 & & & & & & \\
\hline $\mathrm{ROE}^{4}$ & 19,5 & 14,6 & 10,6 & 13,2 & 22,7 & 24,8 & 17,6 \\
\hline N..$^{\circ}$ de empresas & 55 & 96 & 51 & 49 & 48 & 44 & 57 \\
\hline
\end{tabular}

Nota: MMUS\$ significa cantidad en millones de dólares.

$$
{ }^{1} \sigma=0,8^{2} \sigma=2,9^{3} \sigma=1,6^{4} \sigma=5,6
$$

Fuente: Elaboración propia con base en Damodaran (2020). 
El cargo de capital sigue la misma dirección del Ko, salvo en el 2015, y del ANO hasta el 2017. El Ko promedio fue de 7,9 \%, al pasar del costo de capital más bajo en el 2017 (6,8 \%) al más alto en el 2018 (8,8 \%). Por su parte, el ANO promedio fue de \$109.374, que comenzó el periodo con \$ 107.259 MMUS y lo finalizó con el monto más alto en el 2019 (\$ 115.413 MMUS).

La creación de valor se puede comprobar en unidades monetarias, al observar que la UODI superó al cargo de capital anualmente, y en términos porcentuales, al probar que el coeficiente de la relación UODI/ANO excedió el Ko año a año. Como resultado, queda un porcentaje de utilidad residual promedio de 5,0 \% (la diferencia entre 12,9 \% y 7,9 \%).

Durante los seis años el valor económico agregado acumulado fue de \$ 24.384 MMUS a valor presente del primero de enero del 2014, lo que indica que en este sector el objetivo financiero de crear valor económico se cumplió.

\subsection{Desempeño financiero del sector cemento en Colombia (SCC)}

En este apartado se evalúa el ejercicio financiero del sector cementero en Colombia en el periodo 2014-2019 mediante el análisis de su crecimiento, eficiencia, eficacia, efectividad y de su creación (destrucción) de valor económico. 
Tabla 5. Indicadores contables promedios del SCC

\begin{tabular}{|c|c|c|c|c|c|c|c|}
\hline & 2014 & 2015 & 2016 & 2017 & 2018 & 2019 & promedio \\
\hline \multicolumn{8}{|c|}{ Crecimiento (MM\$) } \\
\hline Ventas & 259.768 & 319.244 & 312.621 & 234.299 & 228.654 & 270.418 & 270.834 \\
\hline Activos & 913.658 & 1.071 .542 & 1.130 .858 & 1.049 .966 & 1.051 .252 & 1.083 .061 & 1.050 .056 \\
\hline Utilidad neta & 42.168 & 53.906 & 69.287 & 2.047 & 15.929 & 14.206 & 32.924 \\
\hline \multicolumn{8}{|c|}{ Eficiencia (veces) } \\
\hline Rotación cartera & 6,1 & 7,0 & 7,1 & 4,8 & 4,7 & 5,3 & 5,8 \\
\hline Rotación inventario & 8,5 & 9,4 & 8,8 & 8,3 & 8,7 & 9,0 & 8,8 \\
\hline Rotación activo fijo & 1,4 & 1,2 & 0,8 & 0,6 & 0,6 & 0,7 & 0,9 \\
\hline Rotación activo total ${ }^{1}$ & 0,3 & 0,3 & 0,3 & 0,2 & 0,2 & 0,3 & 0,3 \\
\hline \multicolumn{8}{|c|}{ Márgenes (\%) } \\
\hline Margen bruto & 46,1 & 42,2 & 40,0 & 30,2 & 30,2 & 30,4 & 36,5 \\
\hline Margen operacional & 30,1 & 21,8 & 18,3 & 3,6 & 6,8 & 8,2 & 14,8 \\
\hline Margen neto ${ }^{2}$ & 16,2 & 16,9 & 22,2 & 0,9 & 7,0 & 5,3 & 11,4 \\
\hline \multicolumn{8}{|c|}{ Apalancamiento financiero (\%) } \\
\hline Activo/patrimonio 3 & 144,7 & 152,7 & 154,9 & 157,1 & 161,8 & 162,7 & 155,6 \\
\hline \multicolumn{8}{|c|}{ Efectividad (\%) } \\
\hline ROA & 8,7 & 7,0 & 5,2 & 0,8 & 1,5 & 2,1 & 3,9 \\
\hline $\mathrm{ROE}^{4}$ & 6,8 & 8,3 & 9,7 & 0,3 & 2,5 & 2,2 & 4,7 \\
\hline
\end{tabular}

Nota: MM\$ significa cantidad en millones de COP

$$
{ }^{1} \sigma=0,04{ }^{2} \sigma=8,2^{3} \sigma=6,6^{4} \sigma=3,8
$$

Fuente: elaboración propia con base en EMIS Professional (2020). 
Los indicadores de crecimiento presentaron diversas orientaciones en el sexenio: las ventas subieron al comienzo y final del periodo, pero bajaron en los años intermedios, y los activos crecieron continuamente ${ }^{1}$, con un bache en el 2017. Entretanto, la utilidad neta subió hasta 2016, aunque luego fluctuó. Las medias de las ventas, activos y utilidad neta fueron en su orden de \$270.834 MM, \$ 1.050.056 MM y \$ 32.924 MM (parte a de la tabla 5).

Los indicadores de eficiencia solo muestran similitudes en su comportamiento en el año 2017 cuando caen, y en el 2019 cuando ascienden; en el resto de los años sus orientaciones son distintas. La rotación de cartera tuvo altibajos bianuales hasta el 2019, rotando alrededor de 5,8 veces al año, con lo cual mostró una mejor eficiencia en el 2016 (7,1 veces) y una peor eficiencia en 2018 (4,7 veces). Por su parte, la rotación de inventario varió con frecuencia bianual entre 2016 y 2019, cercano a una media de 8,8 veces, y logró una mejor gestión en el 2015 (9,4 veces), pero un mayor deterioro de ésta en el 2017 (8,3 veces). Entretanto, la rotación del activo fijo disminuyó desde comienzo del periodo (1,4 veces) hasta el 2017 (o,6 veces), se estabilizó en el 2018, pero en el 2019 aumentó, con lo que rotó alrededor de 0,9 veces al año. En cambio, la rotación del activo total se mantuvo estable en o,3 veces hasta el 2016, bajó a 0,2 veces entre 2017-2018, pero en el 2019 sube a 0,3 veces. $\mathrm{Su}$ rotación promedio fue de 0,3 veces al año (parte b de la tabla 5). En promedio fue mayor la rotación de inventarios, seguida de rotación de cartera y, posteriormente, de la rotación del activo fijo.

\footnotetext{
1 "Las empresas realizaron millonarias inversiones entre 2015 y 2016 en nuevos centros de producción, como la planta de Cemex en Maceo, Antioquia, por US\$ 420 millones, la planta de Corona y la de la compañía de origen español, Molins, en Sonsón, por US\$238 millones" (Sectorial 2018, p. 9).
} 
El comportamiento de los indicadores de eficacia fue diferente, menos en el año 2017, cuando descendieron; además, siempre fueron positivos. El margen bruto cayó de 46,1 \% (registrado en 2014) a 30,2 \% (reportado en el 2017 y 2018). En este lapso redujo su eficacia en el control de costos de ventas; en el último año volvió a subir este margen a 30,4\%.

Simultáneamente, el margen operacional descendió de un 30,1 \% logrado en 2014 a un 3,6 \% obtenido en el 2017; efectivamente, en este tiempo disminuyó su eficacia en el control de costos y gastos operacionales, pero volvió a aumentar este margen en los dos años siguientes, hasta llegar a un $8,2 \%$ en el año 2019. Por su parte, el margen neto subió de un 16,2 \%, conseguido en el 2014, a un 22,2 \% que obtuvo en el 2016, por lo que aumentó al máximo su eficacia en el control de todas las erogaciones en estos tres años, pero al siguiente año se derrumbó al llegar colocar este margen en un $0,9 \%^{2}$. Finalmente, no alcanzó a ser tan eficaz como en el primer trienio, aunque en los dos años siguientes lo aumentó con altibajos.

Al comparar el margen bruto promedio (36,5\%) con el margen operacional promedio (14,8 \%) y con el margen de utilidad neta promedio $(11,4 \%)$, se revela que los costos de ventas representaron el 63,5\%, las erogaciones operacionales el $21,7 \%$ y el neto de actividades no operacionales el 3,4\% (parte c de la tabla 5).

El apalancamiento financiero subió en el transcurso del sexenio, pues pasó de 144,7 \% en el 2014 a 162,7 \% en el 2019, y dejó un promedio de 155,6 \% (parte d de la tabla 5).

\footnotetext{
${ }^{2}$ Las demoras en la ejecución de obras 4G y los bajos índices de comercialización de vivienda nueva fueron los dos principales factores que le impidieron a las cementeras construir un buen 2017. De acuerdo con los datos de las 1000 empresas que entregó la Supersociedades, las cinco compañías más grandes del mercado cerraron en terreno negativo: mientras que la ganancia de cada firma cayó más de 70 \%, las ventas lo hicieron en 10 \%" (Bohórquez, 2018).
} 
Los indicadores de efectividad fueron positivos, pero solo presentaron comportamientos afines en el bienio 2017-2018.

El ROA se redujo de 8,7\% en el 2014 a 0,8 \% en el 2017, pero luego aumentó hasta llegar a 2,1\% en el 2019; en el sexenio sigue la misma tendencia del margen operacional. El ROE aumentó de 6,8 \% en el 2014 a 9,7\% en el 2016, pero luego varió sin sobrepasar los indicadores del primer trienio. En los seis años sigue la misma orientación del margen neto.

El ROA promedio del sexenio fue de 3,9\%, producto del margen del operacional (14,8 \%) y la rotación de activos totales (o,3 veces). Mostró una mayor efectividad en el logro de beneficios para la empresa en el 2014 $(8,7 \%)$, cuando el margen operacional fue más alto (30,1\%), y uno de los años con mayor rotación del activo total (o,3 veces). Se evidenció una menor efectividad para los inversionistas en el 2017 (o,8 \%), cuando el margen operacional fue más bajo (3,6 \%), y uno de los dos años con menor rotación del activo total ( 0,2 veces).

Por lo tanto, se puede inferir que el comportamiento de la efectividad en el logro de beneficios para la empresa cementera dependió más de la eficacia en la gestión de erogaciones operacionales que de la eficiencia en la utilización de activos, que en muchos años permaneció invariable, ante los cambios del ROA.

El ROE promedio fue de 4,7\%, producto del margen neto (11,4 \%), la rotación de activos totales ( 0,3 veces) y el apalancamiento financiero (155,6 \%). Esto revela una mayor efectividad en la obtención de beneficios para los propietarios en el 2016 (9,7\%), cuando el margen neto fue mayor $(22,2 \%)$ y era uno de los años con la rotación del activo más alta $(0,3$ veces), pero no con el mayor apalancamiento financiero (154,9\%). También refleja una menor efectividad para los dueños en el 2017 (o,3 \%), 
cuando el margen neto fue menor (o,9 \%) y uno de los años de menor rotación del activo ( 0,2 veces), aun cuando el apalancamiento financiero no fue el menor $(157,1 \%)$.

Lo anterior permite deducir que el comportamiento de la efectividad en el logro de utilidades para los socios obedeció principalmente a la orientación de la eficacia en la gestión de erogaciones totales, que fue aumentada por el apalancamiento financiero. Mientras, la incidencia de la eficiencia en el uso del activo sobre el comportamiento de la efectividad para los dueños fue menor.

El ROE promedio (4,7 \%) fue mayor al ROA promedio (3,9\%) porque contó con apalancamiento financiero positivo en todos los años, lo que contrarrestó un margen operacional promedio superior al margen neto promedio.

La empresa promedio colombiana del sector cemento destruyó valor en los últimos cuatro años de los seis evaluados, lo que dejó como promedio anual un EVA de \$ -6.920 MM. El sexenio inició con el mayor EVA positivo ( $\$ 20.445 \mathrm{MM}$ ), pero a partir del siguiente año comenzó a disminuirse (\$10.054 MM) hasta presentar EVA negativos crecientes entre 2016 (\$ -7.026 MM) y 2017 (\$ -29.393 MM); en los dos años sucesivos empezó a recuperarse, pero dentro de una franja negativa: \$ 19.012 MM en el 2018 y \$-16.587 MM en el 2019.

El EVA sigue la misma orientación de la UODI, excepto en el 2015, y contraria al cargo de capital, salvo en el 2017 y 2019; en promedio los accionistas anhelaban ganar al menos $\$ 31.173 \mathrm{MM}$, pero tan solo lograron \$24.253 MM, teniendo que asumir una destrucción de valor en el periodo (tabla 6). 
Los indicadores de UODI se muestran en la sección a de la tabla 6: la UAII y los impuestos mostraron una relación positiva con la UODI. El promedio de UAII fue de \$32.956 MM y el de los impuestos fue de \$ 8.703 MM. 
Tabla 6. EVA promedio por empresa y sus inductores del SCC

\begin{tabular}{|c|c|c|c|c|c|c|c|}
\hline & 2014 & 2015 & 2016 & 2017 & 2018 & 2019 & promedio \\
\hline EVA (MM\$) & 20.445 & 10.054 & -7.026 & -29.393 & -19.012 & -16.587 & $-\$ 6.920$ \\
\hline UODI (MM\$̧) & 37.568 & 47.903 & 37.094 & 2.523 & 8.427 & 12.004 & 24.253 \\
\hline Cargo de capital (MM\$̧) & 17.123 & 37.849 & 44.120 & 31.916 & 27.438 & 28.592 & 31.173 \\
\hline \multicolumn{8}{|c|}{ a. Indicadores del inductor UODI } \\
\hline UAII (MM\$) & 50.091 & 63.871 & 49.458 & 3.822 & 12.577 & 17.917 & 32.956 \\
\hline Impuestos (MM\$) & 12.523 & 15.968 & 12.365 & 1.300 & 4.150 & 5.913 & 8.703 \\
\hline \multicolumn{8}{|c|}{ b. Indicadores del inductor cargo de capital } \\
\hline ANO (MM\$) & 231.881 & 371.877 & 401.433 & 386.406 & 379.005 & 397.155 & 361.293 \\
\hline Ko (\%) ${ }^{1}$ & 7,4 & 10,2 & 11,0 & 8,3 & 7,2 & 7,2 & 8,5 \\
\hline \multicolumn{8}{|c|}{ c. Indicadores del inductor ANO } \\
\hline KTNO (MM\$̦) & 102.105 & 77.019 & 69.195 & 51.725 & 43.640 & 51.030 & 65.786 \\
\hline AFNO (MM\$) & 129.776 & 294.858 & 332.238 & 334.681 & 335.365 & 346.124 & 295.507 \\
\hline \multicolumn{8}{|c|}{ d. Indicadores del Inductor Ko } \\
\hline $\operatorname{Ke}(\%)$ & 16,2 & 20,7 & 15,8 & 14,0 & 14,5 & 14,8 & 16,0 \\
\hline Ki $(\%)^{2}$ & 7,2 & 8,0 & 11,4 & 9,8 & 7,8 & 7,4 & 8,6 \\
\hline L (\%) & 81,8 & 71,5 & 66,4 & 76,3 & 78,1 & 77,4 & 75,2 \\
\hline$t(\%)$ & 25,0 & 25,0 & 25,0 & 34,0 & 33,0 & 33,0 & 29,2 \\
\hline \multicolumn{8}{|c|}{ e. Sistema Dupont del índice UODI/ANO } \\
\hline UODI/ANO (\%) ${ }^{3}$ & 16,2 & 12,9 & 9,2 & 0,7 & 2,2 & 3,0 & 7,4 \\
\hline UODI/Ventas (\%) & 14,5 & 15,0 & 11,9 & 1,1 & 3,7 & 4,4 & 8,4 \\
\hline Ventas/ANO (veces) & 1,1 & 0,9 & 0,8 & 0,6 & 0,6 & 0,7 & 0,8 \\
\hline
\end{tabular}

Revista Activos

ISSN: 0124-5805 | e-ISSN: 2500-5278 | DOI: https://doi.org/10.15332/25005278

Vol. 19 N. ${ }^{\circ} 1$ | enero-junio de 2021 


\begin{tabular}{|l|c|c|c|c|c|c|c|}
\hline & 2014 & 2015 & 2016 & 2017 & 2018 & 2019 \\
\hline & \multicolumn{3}{|c|}{ f. Valor del mercado agregado } \\
\hline VMA a 01-01-2014 (MM\$) & -21.109 & & \\
\hline
\end{tabular}

Notas:

MM\$ significa cantidad en millones de COP.

$1 \sigma=1,6 \%$.

${ }^{2}$ Tasa promedio anual de crédito corporativo o preferencial proporcionado por la Superintendencia Financiera de Colombia (2020).

$3 \sigma=6,4 \%$.

Fuente: elaboración propia con base en EMIS Professional (2020), Superintendencia Financiera de Colombia (2020) y

Damodaran (2020). 
Los inductores del cargo de capital se exhiben en la sección b de la tabla 6: el ANO y el Ko mantiene la misma tendencia hasta el penúltimo año, que es similar a la del cargo de capital; en el 2019 el ANO aumenta y el Ko se mantiene invariable. El promedio del ANO fue de \$361.293 MM y del Ko $8,5 \%$.

Los indicadores del inductor ANO se presentan en la parte c de la tabla 6: el KTNO se redujo hasta el penúltimo año, y creció al final, mientras que el AFNO aumentó en el sexenio. La combinación de ambos determinó el comportamiento del ANO. El AFNO representó el 81,8 \% del ANO: los promedios del AFNO y el KTNO fueron, en su orden, de \$295.507 MM y de $\$ 65.786 \mathrm{MM}$.

Los inductores de Ko se reportan en la sección d de la tabla 6, de modo que se observa una relación directa entre Ko y Ki y una relación inversa entre Ko y L hasta el penúltimo año. El Ki fue inferior al Ke en cada año. Los promedios de estos indicadores fueron: $\mathrm{Ke}=16,0 \%, \mathrm{Ki}=8,6 \%$; $=$ $75,2 \%$ y t $=29,2 \%$.

El EVA y Ko mantuvieron una relación inversa salvo en dos años: 2017 y 2019; mientras que el EVA y el indicador UODI/ANO conservaron una relación directa.

El promedio del rendimiento después de impuestos del ANO (7,4 \%) fue inferior al promedio del Ko $(8,5 \%)$, La diferencia genera una pérdida residual del 1,1 \% que confirma la razón por la cual este sector destruyó valor en el sexenio.

Los indicadores que determinan la UODI/ANO se reportan en la parte e de la tabla 6, con lo cual se encuentra una relación positiva entre UODI/ANO y UODI/ventas a partir del 2016, que cambia de orientación cada dos años; y una relación directa entre UODI/ANO y ventas/ANO, salvo en el 2018, que desciende hasta 2017, se estanca en el 2018, pero vuelve a subir en el 
2019. Este comportamiento muestra que, cuando se trata del rendimiento después de impuestos del ANO, la eficacia en la gestión de erogaciones operacionales sigue dominando la tendencia, aunque con una mayor participación de la eficiencia en el uso de los activos netos operacionales que como lo hacía la gestión de todos los activos ${ }^{1}$.

El VMA del sector cemento en Colombia al 1 de enero de 2014 fue de \$21.109 MM, lo que corrobora que durante el sexenio no se cumplió el propósito financiero de crear valor, a pesar de que los indicadores contables de desempeño habían mostrado utilidades, márgenes y rendimientos positivos (parte f de la tabla 6 y parte a, c y e, de la tabla 5).

\section{Análisis y contrastes de los resultados}

En esta sección se cotejan los indicadores de desempeño financiero que miden el crecimiento, la eficiencia, la eficacia, la efectividad y el valor económico agregado del sector cemento en Colombia (SCC) con los datos conseguidos de las ediciones especiales de la revista Dinero entre el 2014 y el 2019 de las grandes compañías del sector cemento y concreto en Colombia (GCC) y del sector suministros de construcción en los Estados Unidos de América (SCEUA).

\section{Crecimiento}

Las ventas, activos y utilidad neta promedios del SCC fueron inferiores a los de las GCC, como también en cada año, salvo la utilidad neta en el 2014 y 2017, debido a que este último representa al colectivo de empresas de mayor tamaño en Colombia. La orientación de estos indicadores fue semejante en ambos grupos hasta el 2018, salvo las ventas en el 2016. En

\footnotetext{
${ }^{1}$ Cuando se analizó el ROA con el sistema Dupont se encontró el escaso impacto de la eficacia (rotación de activos totales) sobre su comportamiento.
} 
general todos los indicadores subieron hasta el 2016 (salvo una caída en las ventas de SCC en el 2016), en el 2017 disminuyeron, en el siguiente año aumentaron (menos las ventas que disminuyeron), en el último año los indicadores del SCC subieron (exceptuando la utilidad neta, la cual disminuyó) y los de las GCC bajaron (parte a de las tablas 3 y 5).

Al comparar 2014 con 2019 se observa que las utilidades netas disminuyeron un $66,3 \%$ en el SCC y un $63,9 \%$ en las GCC, mientras que las ventas aumentaron un 4,1 \% en el SCC y un 10,2 \% en las GCC; no obstante, los activos subieron un 18,5\% en el SCC y bajaron un 2,5\% en las GCC.

\section{Eficiencia}

La rotación anual y promedio del sexenio de los activos totales fue inferior en el SCC; además, la variabilidad de este indicador fue mayor en el SCC ( $\sigma$ $=0,04$ veces vs $\sigma=0,02$ veces en las GCC). A partir de esto, se puede deducir que las empresas de mayor tamaño de este sector fueron más eficientes en el uso de los recursos físicos, y esa eficiencia fue más estable (parte b y nota 1 de las tablas 3 y 5). Esto significa que cada unidad monetaria invertida en activos genera menos ventas en todo el sector cementero y de forma más dispersa que la que se produce en las grandes empresas de este sector.

\section{Eficacia}

El margen de utilidad neta anual y promedio en el sexenio fue superior en el SCC, aunque con una mayor variabilidad ( $\sigma=8,2 \%$ vs $\sigma=4,4 \%$ en las GCC). Los márgenes de utilidad neta fueron positivos cada año (parte c y nota 2 de las tablas 3 y 5 ). Con esto, se puede deducir que las empresas de mayor tamaño fueron menos eficaces, aunque más estables, en el control de costos y gastos que todo el sector cementero; dicho de otra forma, las 
ventas del sector cementero producen más utilidad neta y de forma más dispersa que las que originan el colectivo de las empresas más grandes de este sector.

\section{Apalancamiento financiero}

$\mathrm{El}$ apalancamiento financiero anual y promedio fue menor que la de las GCC, y con menor inestabilidad ( $\sigma=6,6 \%$ versus una $\sigma=11,9 \%$ en las GCC); esto permite inferir que el SCC usó una proporción de deuda más baja y estable en comparación con las GCC (parte d y nota 3 de las tablas 3 y 5). El menor apalancamiento financiero y su menor dispersión redujo su riesgo por deuda frente al grupo de empresas más grandes del sector cementero.

\section{Efectividad}

$\mathrm{Al}$ contrastar el ROE promedio de las GCC $(4,4 \%)$ con el del SCC $(4,7 \%)$ y el del SCEUA (17,6 \%), se aprecia una considerable inferioridad de los dos grupos colombianos, que se corroboró cada año, aunque con una mayor estabilidad de los primeros ( $\sigma=3,1 \%$ en las GCC y $\sigma=3,8 \%$ en el SCC, versus $\sigma=5,6 \%$ en el SCEUA) (parte e y nota 4 de las tablas 3 y 5 , y tabla 4 con su nota 4 ).

$\mathrm{Al}$ cotejar el ROE anual y promedio de las dos agrupaciones colombianas, se halló que fue superior en el SCC, excepto en el 2015 y 2018; esto se debió a que el margen neto fue mayor en el SCC (en promedio 11,4 \% frente a un 5,8 \% en las GCC), con lo que contrarrestó su menor rotación de activos y apalancamiento financiero ( 0,3 veces y $155,6 \%$ versus 0,4 veces y 177,6 \% en las GCC respectivamente), aunque con una mayor inestabilidad del ROE en el SCC ocasionado a la mayor desviación estándar que presentaron su margen neto y su rotación de activos (tablas 2 y 4). Es decir, la efectividad en el logro de utilidades fue mayor para los 
propietarios de todo sector el cementero que para los propietarios de las grandes empresas de este sector porque las ventas produjeron más utilidades netas, que compensaron con creces las menores ventas que generaron los activos y los beneficios de un menor apalancamiento financiero positivo.

Los resultados indican que la efectividad en el logro de beneficios para los dueños de la empresa promedio del sector cementero en Colombia fue mayor que la de la gran empresa de este sector en el periodo 2014-2019, suscitado por su mejor eficacia en el control de erogaciones, aunque con una mayor inestabilidad.

\section{Valor económico agregado}

El EVA del SCC fue solo positivo en los dos primeros años, en cambio en el SCEUA fue positivo cada año², además, sus orientaciones fueron similares, salvo el 2017. Al repasar los inductores se encontró que el promedio de UODI/ANO en el SCC (7,4 \%) fue inferior al del SCEUA (12,9\%), pero el promedio del costo de capital en el SCC $(8,5 \%)$ fue superior al del SCEUA $(7,9 \%)$, produciendo un porcentaje de pérdida residual en el SCC $(-1,1 \%)$ y un porcentaje de utilidad residual en el SCEUA (5,0 \%) (tablas 4 y 6).

Los inductores del EVA fueron más inestables en el SCC como se puede reafirmar al contraponer la $\sigma$ del indicador UODI/ANO $(6,4 \%$ en el SCC vs 2,9\% en el SCEUA), y la $\sigma$ del Ko (1,6 \% en el SCC vs o,8 \% en el SCEUA).

El SCEUA crea valor porque la rentabilidad del activo neto operacional supera al costo de capital cada año, situación que solo acontece en el SCC en dos años, lo que muestra que la efectividad en el logro de utilidades del SCC no compensa los beneficios requeridos por los propietarios.

\footnotetext{
${ }^{2}$ El VMA del sexenio para el SCC fue de \$-21.109 MM, y para el SCEUA fue de US\$24.384 MM. El año crítico para el SCC fue el 2017 cuando se destruyó un cuantioso valor.
} 


\section{Hallazgos e implicaciones}

Los resultados de esta investigación muestran que el sector cementero en Colombia destruyó valor en el periodo 2014-2019 porque el promedio de la utilidad operacional después de impuestos fue inferior al promedio del costo de sus recursos financieros. Esta es una señal que va en contravía a lo mostrado por los indicadores contables que revelaron resultados favorables. No obstante, gran parte de esta destrucción de valor obedeció a la baja efectividad para generar utilidades a los inversionistas, por la reducida eficacia en el control de erogaciones que se presentó en 2017. También se pudo encontrar que el SCC fue más efectivo que las GCC, por ser más eficaz en el control de costos y gastos. No obstante, el sector norteamericano afín fue más efectivo que los dos grupos colombianos, y creó valor económico agregado; esto ocurrió no solo porque la rentabilidad de la inversión fue más alta, sino también, porque el costo de los recursos fue más bajo.

Esta investigación solo abarcó los factores esenciales que incidieron sobre el desempeño financiero del sector cemento en Colombia en el periodo 2014-2019. Queda pendiente por evaluar otras variables que influyen sobre el desempeño y competitividad financiera, como, por ejemplo, el tamaño, la edad, su naturaleza jurídica, entre otros, que pueden ofrecer resultados complementarios a los ofrecidos por este estudio.

\section{Conclusiones}

La industria de cemento, cal y yeso en Colombia tuvo una considerable participación en la generación de empleo y producción durante el periodo 2014-2019. En este estudio se encontró un aumento de sus ventas, activos y una disminución de su utilidad neta, aunque con variaciones; este mismo 
comportamiento se presentó en la gran empresa de este sector, pero con disminución en los activos.

Los rendimientos anuales de los activos en el SCC fueron positivos y su orientación dependió más de la eficacia en el monitoreo de erogaciones operacionales que de la eficiencia en la administración de activos. Además, los rendimientos anuales del patrimonio también fueron positivos, y estuvieron mayormente influenciados por la eficacia en el control de las erogaciones totales, que fue amplificada por el apalancamiento financiero.

En el sexenio resultó ser más efectivo el SCC que las GCC, dado que el ROE promedio fue de 5,0 \% para el primero y 4,4 \% para la segunda; esto sucedió porque el SCC fue más eficaz en el control de todos los costos y gastos (margen de utilidad neta), que fue suficiente para compensar su menor eficiencia en la utilización de sus recursos (rotación de activos) y el menor uso de un apalancamiento financiero positivo. No obstante, el ROE fue más inestable en el SCC, lo que implicó un mayor riesgo.

Al compararse con el SCEUA, se detectó que este último fue más efectivo que las dos agrupaciones nacionales, dado que su ROE fue superior, aunque más inestable.

Los resultados favorables que mostraron los indicadores contables de desempeño financiero del sector cementero en Colombia no fueron confirmados por los indicadores de gestión de valor, pues se encontró que este sector destruyó valor económico agregado en cuatro años del sexenio, siendo considerable la pérdida residual del año 2017, que dejó como resultado un VMA negativo. Esto fue causado porque la rentabilidad promedio del activo neto operacional después de impuestos fue inferior al promedio del costo de capital. El comportamiento del EVA siguió la dirección del rendimiento después de impuestos del activo neto operacional, y este a su vez se vio más influenciado por el margen de UODI 
que por la rotación de ANO. Lo anterior confirmó la relevancia de la eficacia en el control de costos y gastos sobre el desempeño de las empresas de este sector.

Estos resultados difieren con los del SCEUA, donde se creó valor cada año.

El sector cemento en Colombia no alcanzó a cumplir con el objetivo financiero de crear valor de mercado agregado en el periodo, primordialmente porque ha visto deteriorada su efectividad en lograr beneficios a sus accionistas en los últimos años, dada la reducción de su eficacia en el control de erogaciones, que tocó fondo en el 2017. Este inductor puede mejorar si implementa estrategias que lo vuelvan a colocar en una posición al menos similar a los primeros años de este sexenio cuando creó valor económico agregado.

\section{Referencias}

Anaya, H. (2018). Análisis financiero aplicado, bajo NIIF (16. ${ }^{\mathrm{a}}$ ed.). Universidad Externado de Colombia.

Atrill, P. (2017). Financial management for decision maker (8. ${ }^{a}$ ed.). Pearson Educación.

Brealey, R., Myers, S. y Allen, F. (2015). Principios de finanzas corporativas (11. ${ }^{\mathrm{a}} \mathrm{ed}$. .). McGraw- Hill.

Brigham, E. y Ehrhardt, M. (2018). Finanzas corporativas. Enfoque central (1. $\left.{ }^{\mathrm{a}} \mathrm{ed}.\right)$. Cengage Learning.

Damodaran, A. (2020). Data Damodaran Online [base de datos]. http://pages.stern.nyu.edu/ adamodar/

Departamento Administrativo Nacional de Estadística. (2020a). Encuesta anual manufacturera [anexos principales variables 2014-2018]. https://www.dane.gov.co/index.php/estadisticas-por-tema/industria/encuestaanual-manufacturera-enam/eam-historicos 
Departamento Administrativo Nacional de Estadística. (2020b). Encuesta anual manufacturera [anexos principales variables 2019].

https://www.dane.gov.co/index.php/estadisticas-por-tema/industria/encuestaanual-manufacturera-enam

Departamento Administrativo Nacional de Estadística. (2020c). Clasificación industrial internacional uniforme de todas las actividades económicas. Revisión 4 adaptada para Colombia. CIIU Rev. 4 A.C.

https://www.dane.gov.co/files/sen/nomenclatura/ciiu/CIIU Rev 4 AC2020.pdf

Departamento Administrativo Nacional de Estadística. (2021). Anexos estadísticos de PIB producción. PIB a precios constantes 2021.

https://www.dane.gov.co/index.php/estadisticas-por-tema/cuentasnacionales/cuentas-nacionales-trimestrales/pib-informacion-tecnica

Dumrauf, G. (2017). Finanzas corporativas: un enfoque latinoamericano (3. $\left.{ }^{\mathrm{a}} \mathrm{ed}.\right)$. Alfaomega.

EMIS Professional (2020). ISI Emerging Markets Group (Base de datos). https://wwwemis-com.bd.univalle.edu.co/php/dashboard?

Gitman, L. y Zutter, C. (2016). Principios de administración financiera (14. ${ }^{\mathrm{a}}$ ed.). Pearson Educación.

Modigliani, F. \& Miller, M. (1963). Corporate income taxes and the cost of capital: a correction. The American Economic Review, 53, 433-443.

Neira, L. (2019, 5 de noviembre). Desde 2010 la capacidad instalada de las plantas ha crecido pasando de 6,1 millones de toneladas a 8,4 millones, según el Dane. La República. https://www.larepublica.co/empresas/en-la-ultima-decada-haningresado-seis-nuevas-cementeras-al-mercado-local-2928246

Dinero. (2014, 13 de junio). 5000 empresas, 447, 230.

Dinero. (2015, 12 de junio). Ranking 5ooo empresas, 471, 194.

Dinero. (2016, 10 de junio). Las 5000 empresas, 495, 176.

Dinero. (2017, 21 de julio). Ranking 5ooo empresas, 522, 136.

Dinero. (2018, 21 de junio). Ranking 50oo empresas, 544, 140.

Dinero. (2019, 28 de junio). Ranking 5ooo empresas, 568, 138-140. 
Dinero. (2020, septiembre 18). Ranking 50oo empresas, 598, 108-110.

Rivera, J. (2017). Introducción a la administración financiera: fundamentos y aplicaciones para crear valor. Universidad del Valle.

Rivera, J. y Alarcón, D. (2012). El cargo de capital en la evaluación del desempeño financiero de empresas innovadoras de confecciones de Cali. Estudios Gerenciales,38(123),85-100. https://doi.org/10.1016/s0123-5923(12)70206-1

Ross, S., Westerfield, R., Jaffe, J. y Jordan, B. (2019). Corporate finance (12. ${ }^{\mathrm{a}}$ ed.). McGraw-Hill Education.

Salaga, J., Bartosova, V. y Kicova, E. (2015). Economic value added as a measurement tool of financial performance. Procedia Economics and Finance, 26, 484-489. https://doi.org/10.1016/S2212-5671(15)00877-1

Sectorial (2018, junio). Informe sector cemento. Recuperado de https://www-emiscom.bd.univalle.edu.co/php/search/docpdf?pc $=$ CO\&sv $=$ EMIS\&doc id $=63502562$ $\underline{8}$

Sectorial (2020, junio). Informe sector cemento. Recuperado de https://www-emiscom.bd.univalle.edu.co/php/search/docpdf?pc $=$ CO\&doc id $=682354804$

Stern, J. y Willett, J. (2014). A look back at the beginnings of EVA and value based management: An interview with Joel M. Stern. Journal of Applied Corporate Finance, 26(1), 39-46. https://papers.ssrn.com/sol3/papers.cfm?abstract id=2422149

Stewart, B. (2000). En busca del valor. Ediciones Gestión 2000.

Superintendencia Financiera de Colombia. (2020). Tasa de interés y desembolsos por modalidad de crédito. https://www.superfinanciera.gov.co/jsp/loader.jsf?1Servicio=Publicaciones\&lTipo =publicaciones\&lFuncion=loadContenidoPublicacion\&id=60955

Worthington, A. y West, T. (2001). Economic value-added: A review of the theoretical and empirical literature. Asian Review of Accounting, 9(1), 67-86.

https://papers.ssrn.com/sol3/papers.cfm?abstract id=2169807 
Anexo 1. Empresas de la industria del cemento, cal y yeso en Colombia

\begin{tabular}{|c|c|c|c|}
\hline NIT & Razón social & NIT & Razón social \\
\hline 890100251 & Cementos Argos S.A. & 800178319 & $\begin{array}{c}\text { Procesadora De Cales Procecal } \\
\text { S.A.S }\end{array}$ \\
\hline 860002523 & Cemex Colombia S.A. & 900882921 & Productora de Cemento S.A.S. \\
\hline 860009808 & Holcim (Colombia) S.A. & 900646912 & Caltek S.A.S. \\
\hline 900164755 & Zona Franca Argos S.A.S. & 830120172 & Cementos Atlas S.A. \\
\hline 900570964 & Ultracem S.A.S. & 900889452 & Minerales Prodeka S.A.S. \\
\hline 900233101 & Cementos San Marcos S.A.S & 900803391 & Cementos Nacionales S.A. \\
\hline 890304607 & Impadoc S.A. & 890800127 & Cementos de Caldas S.A. \\
\hline 900657956 & Molienda de La Sabana S.A.S. & 890207056 & $\begin{array}{l}\text { Industria Yesera Santandereana } \\
\text { Ltda }\end{array}$ \\
\hline 824004657 & Cementos y Calizas de la Paz S.A. & 890600139 & $\begin{array}{l}\text { Esteban Montana y Compañia } \\
\text { Limitada }\end{array}$ \\
\hline 800256143 & Empresa de Agregados Calcareos S.A.S. & 900907364 & $\begin{array}{l}\text { Empresa Colombiana de } \\
\text { Cementos S.A.S. }\end{array}$ \\
\hline 900428195 & Lhoist Colombia S.A.S. & 900250765 & Prodecales S.A.S. \\
\hline 811027220 & Cales De Colombia S.A. & 900428871 & Cales Fenix S.A.S. - Liquidacion \\
\hline 890910599 & Productos Minerales Calcareos S.A. & 890900120 & Suministros De Colombia S.A.S. \\
\hline 830120480 & Cementos del Oriente S.A. & & \\
\hline
\end{tabular}

Fuente: elaboración propia con base en EMIS professional (2020).

Revista Activos

ISSN: 0124-5805 | e-ISSN: 2500-5278 | DOI: https://doi.org/10.15332/25005278

Vol. 19 N. ${ }^{\circ} 1$ | enero-junio de 2021 